4. A. B. Migdal. Sov. Phys. JETP 16 (1964) 1366.

5. G. M. Eliashberg. Sov. Phys. JETP 14 (1963) 886.
6. J. Gavoret and P. Nozières, Ann, of Phys, $28,349$.

7. H. Winter, Z. Phys., to be published.

\title{
EVIDENCE FOR A SUPERFLUID-INDUCED SURFACE BARRIER FOR ELECTRONS IN LIQUID HELIUM
}

\author{
W. SCHOEPE \\ Physik-Department der Technischen Hochschule Miinchen, Germany
}

and

\section{PROBST}

Zentralinstitut für Tieftemperaturforschung der Bayerischen Akademie der Wissenschaften

Garching bei Minchen, Germany

Received 24 March 1970

\begin{abstract}
The free surface of liquid helium acts as an energy barrier on electron currents across the surfac into the vapor, if the liquid is superfluid. In zero electric field, the barrier vanishes at $T_{\lambda}$. The measurements were performed in pure ${ }^{4} \mathrm{He}$ and in a solution of ${ }^{3} \mathrm{He}$ in ${ }^{4} \mathrm{He}$.
\end{abstract}

Bruschi et al. [1] have reported that for temperatures less than $1.7^{\circ} \mathrm{K}$ electrons encountered increasing difficulty to pass through the free surface of liquid ${ }^{4} \mathrm{He}$ into the vapor phase, if the temperature is lowered. Below $1.5^{\circ} \mathrm{K}$ the electron current decreased rapidly according to $\exp \left(-\phi / k_{\mathrm{B}} T\right)$, with $\phi / k_{\mathrm{B}}=25 \pm 1^{\circ} \mathrm{K}$. This behavior was mainly attributed to the image-force potential which acts on a charge near the interface of two media having different dielectric constants. The effect of the image-force potential outside the liquid was recently discussed in detail by Cole and Cohen [2]. Using the assumption of the image-force, however, it can be readily shown, that the work function $\phi$ must depend on the extracting field $E$, in contradiction to the results of ref. [1]. In addition, the saturation of the currents at $1.7^{\circ} \mathrm{K}$ remained unexplained since this temperature is much smaller than the barrier. Therefore, we made a special effort to determine $\phi$ with a higher accuracy. Besides pure ${ }^{4} \mathrm{He}$ we also investigated a solution of ${ }^{3} \mathrm{He}$ in ${ }^{4} \mathrm{He}$. Our results demonstrate that the barrier depends not only on the applied field but also on the temperature, giving clear evidence that the barrier is present only in the superfluid state.

The measuring cell was similar to that shown in ref. [1], consisting of three electrodes as indicated in fig. 1 . We measured the currents when the liquid level was above the collector and compared them with the corresponding currents when the liquid surface was below the collector. The currents with the collector being immersed were nearly temperature independent, in agreement with ref. [1]. On the other hand, when the liquid level was lowered below the collector the current showed a striking dependence on the temperature as plotted in fig. 1 for different extracting fields. For example for a field of $154 \mathrm{~V} / \mathrm{cm}$ the current remains temperature independent from $4.2^{\circ} \mathrm{K}$ down to $1.7^{\circ} \mathrm{K}$. Only below $1.70 \mathrm{~K}$ the current decreases rapidly with temperature as $\exp \left(-\phi / k_{\mathrm{B}} T\right)$, indicating an effective energy barrier $\phi / k_{\mathrm{B}}=$ $30.4^{\circ} \mathrm{K}$, which the emitted electrons have to overcome. It is evident that this positive energy barrier only exists well below $1.7^{\circ} \mathrm{K}$ and apparently disappears when this temperature is exceeded. It is essential to point out that for lower extracting fields the energy barrier disappears at higher temperatures, which approach the $\lambda$-point for zero extracting fields. The same behavior is observed in a solution of $32 \%{ }^{3} \mathrm{He}$ in ${ }^{4} \mathrm{He}$ below the shifted $\lambda$-point of $1.66^{\circ} \mathrm{K}$. Thus a finite energy barrier for the emission of electrons from liquid helium seems to be closely related to the superfluid state.

Besides this temperature dependence of the barrier it is important to emphasize that $\phi$ also 


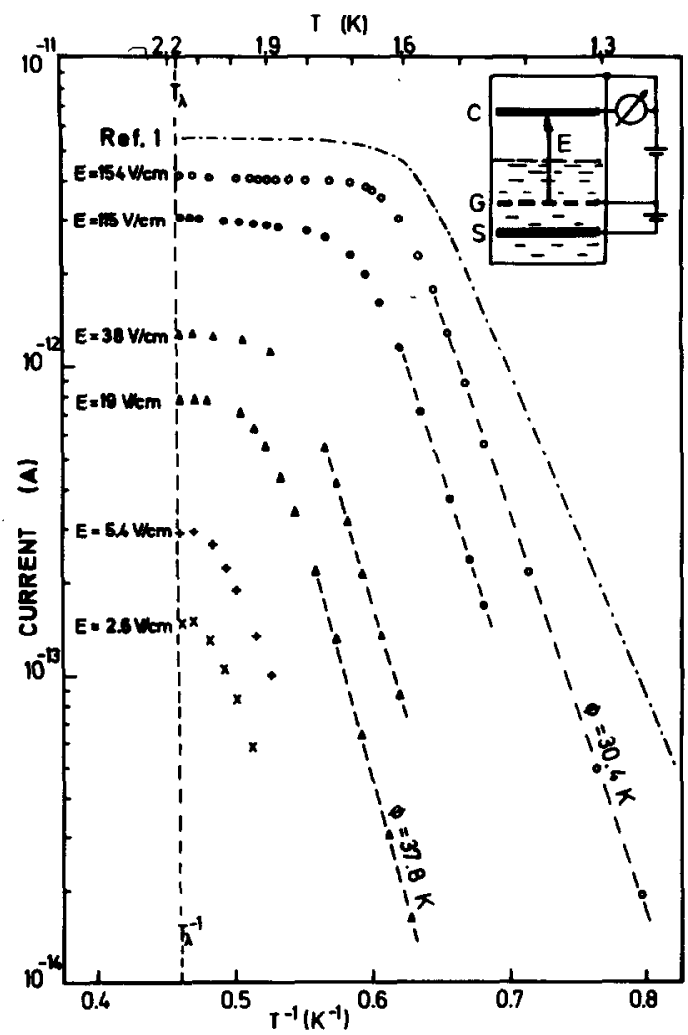

Fig. 1. Measured collector current through the surface of liquid ${ }^{4} \mathrm{He}$ as a function of the temperature at different extracting fields $E$, indicated in the insert together with the electrode structure of the gold plated measuring cell (collector $\mathrm{C}$, grid $\mathrm{G}$, and radioactive source S). The slopes of the dashed curves yield the effective energy barrier $\phi(E)$. The relative current values of ref. [1] are indicated by the dash-dotted curve. depends on the extracting field. This conclusion can be reached not only from the behavior at higher temperatures described above but also from the fact, that at the lower temperatures the slope of the curves in fig. 1 (dashed lines) depends clearly on the extracting field.

A full account of the experimental results including a detailed description of the temperature and field dependence of the surface barrier will be presented elsewhere together with a tentative explanation.

Finally, it should be mentioned that the observed absence of a surface barrier in rotating helium for electrons trapped in vortex lines [3] could now be considered as a possible consequence of the normal fluid vortex core proposed recently by Glaberson, Strayer and Donelly [4].

We thank K. Dransfeld, H. Schmidt and D. Stauffer for advice and helpful discussions. We are also indebted to $W$. Wiedemann for his encouraging interest and to the Deutsche Forschungsgemeinschaft for their financial support.

\section{References}

1. L.Bruschi, B.Maraviglia and F.E.Moss, Phys. Rev. Letters 17 (1966) 682.

2. M.W. Cole and M.H. Cohen, Phys. Rev. Letters 23 (1969) 1238.

3. W. Schoepe and K. Dransfeld, Phys. Letters $29 \mathrm{~A}$ (1969) 165.

4. W.I.Glaberson, D. M.Strayer and R.J.Donnelly, Phys. Rev. Letters 21 (1968) 1780. 